\title{
Vehicle Anti-Theft Tracking System Based on Internet of Thing(IoT)
}

\author{
Utkarsh $^{a}$, Keshav Kumar Jha ${ }^{b}$ \\ ${ }^{a, b}$ Department of Mechanical Engineering , KCC Institute of Technology and Management , Gr Noida ,UP, India
}

\begin{abstract}
Owing to the growth of cars, auto theft has become a major problem in developed countries. A vehicle-anti-theft monitoring(vat) device may then play a critical role in ensuring its safety. Vehicle theft has been a big concern in recent years, and it must be tracked and identified. The vehicle's safety and protection are critical. And if there are several current mechanisms, they all have drawbacks and are expensive. As a result, an effective protection framework is needed. This project identifies auto theft. The feature that links the dc motor to the GPS is the GSM. The location of a vehicle was pinpointed with the aid of the wireless-module ESP 8266 and the GPS as well as GSM communication. a GPS system can accurately point out a vehicle at any climate which is a Satellite-based-navigation system, a GPS device gives the latitude and longitude position.
\end{abstract}

Keywords: GSM Technology, Vehicle Tracking, GPS-GSM, Internet of things (IoT).

\section{INTRODUCTION}

The vehicle crimes result in vehicle theft and trafficking, which affect owners, companies which are responsible for insurance, and public safety in all countries, and have connections to major organized crime networks. In order to prevent vehicle theft, latest systems based on innovative technologies must be implemented to assist law enforcement agency as well as vehicle owners in tracking, controlling, recovering, and arresting thieves. With this presented system, vehicle pursuing, and IOT based control system, which monitors, controls, and proposes clear information about nearby law enforcement agency headquarters that perhaps be analysed rapidly and a reliable mode of transportation.[6]

A networked connection of devices for instance cell phones, sensors, PDAs and additional devices communicates and connect without any human interference with each other is identified as Internet-ofThings (loT). Internet-of-vehicles is the area in which there is communication between vehicles and surroundings the information is shared with the network [2]. Now, IoT also allows conversation and ability to interact between vehicles by using Transducers, gateways as well as actuators.

The current paper is focused on microcontrollers for realtime-vehicle monitoring and IoT technology describing the vehicle-tracking and control system. GPS, GPRS or GSM, and by using microcontroller to incorporate this device, which allows the car to be tracked easily. The device can control and monitor vehicles remotely via SMS control (emergency stop by cutting-off the fuel impeller)[7].
People use vehicle tracking devices as a recovery mechanism and a burglary deterrent. The biggest advantage of vehicle tracking devices is that they provide surveillance by detecting the vehicle's location, and can be used as a deterrent for stolen cars by sending their position coordinates to a police centre as a stolen vehicle warning. When a police station gets a stolen car warning, they will take steps to deter the burglary [10].

It is now used as a supplement or extension to car alarms to protect the vehicle from vandalism, or as a tracking device to track the vehicle in real time. As a result, a variety of applications may be used to disable a car's engine or doors in order to shield the vehicle. Vehicle detection devices have advanced in technologies to the point that they can now identify and monitor illegal vehicle movements and warn the driver. This gives it an edge on other software and bits of hardware that would do the same thing [11].

Vehicle-tracking is most widely used software nowadays. for example, the maps provided to vehicle drivers, can perform a major task in monitoring in addition vehicletracking. Main issue is that car owners will not be able to identify their vehicle in a location due to conflicting maps, which makes tracking and monitoring difficult [1]. It necessitates need of such devices to locate and detect where items were at any given time, as well as the distance travelled on a trip to a car. This may be an extra point to help the police deter robberies and locate the car by drawing on reports from these licensed databases and reviewing and evaluating them [4].

The whereabouts of abandoned cars This machine is a required mechanism for monitoring vehicles if the owner wishes to observe or control them, and it is currently very popular with people who own expensive cars, and it is 
used to prevent vandalism and retrieve stolen vehicles. Using the internet and applications, the collected data can be seen on digital maps [2]. For business processes, entity detection applications are in high demand. The ability to track useful items and properties in real time could solve a variety of problems around the world. The Global Positioning System (GPS) delivers location information under whatever weather situation, both off-line and online. There are many different kinds of GPS tracking devices.

\section{The below are some of the characteristics of this system:}

(1) An Arduino-connected GPS is used to find a vehicle theft,

(2) The vehicle owner's mobile phone receives an alert as a SMS on GSM,

(3) GPS/GSM modules are constantly monitored, and automatically sends an alarm to the owner if they are not working,

(4) The vehicle's location is stored in the IOT cloud platform called thing speak on timely basis.

\section{LITERATURE SURVEY}

A.M. Salman in 2020, [3] conceptualized as well as executed system aimed at conveying essential data for pursuing to reduce the conveyed data between GPSreceiver and the host-server. Module is combining GPS, GSM for conveying data and Arduino-UNO R3 microcontroller. Position coordinates are conveyed to a server by GSM as well as GPRS sends Hipper Text Transfer Protocol requests. The duration between two Hipper Text Transfer Protocol requests was ten seconds when the vehicle moved and no Hipper Text Transfer Protocol requests when the vehicle is stopped. Information is displayed by Google Maps after storing it in the server. This made use of web and mobile applications for tracking and retrieving information.

O. A. Awad as well as D. A. Bahr in 2019 [4] came up with a system to avoid automobile vehicles theft, that is an integrated anti-theft system. This system is based on Raspberry Pi and LTE communication technology Release Module is mounted in the hidden place of automobile. Whenever theft occurs, a call from the proprietor's mobile phone is started to GSM, which Raspberry Pi disconnects as well as trigger GPS to broadcast automobile location information to proprietor as SMS through GSM also possibly monitor it on Google-map, triggering Pi-Camera to take a still image of offender finally sends it to proprietor by superior internet services. proprietor directs regulator signal to halt machine ignition module.
P. Chandra Shreyas et al in 2019 [5] came up with blueprint for tracking and also monitor theft so that owner can identify them. When the offender switches-on his/her vehicle, GPS a Qumulate the variations of entity position as well as embedded photographic-camera takes a still image of the burglar and delivers it to Raspberry Pi. In addition to this, information is stored in cloud, which collects all the data including the data form GSM module as well as is sent to the vehicle's owner. Property proprietor checks the picture and in case of an intruder, he can turn off the vehicle by a special mobile app which can exchange information with cloud in addition get remote access to the automobile.

Tahesin Attar et al in 2018 [6] came up with " Advanced Vehicle System based on IoT" with cloud for providing safety as well as control the theft automatically with insignificant delay time. Cordless communication as well as economical Blue-tooth system. using GSM for messaging. they utilized Infra-Red Sensor, Bluetooth module, Limit Switches, Mobile app as well as used Password entered in key-pad, operates opening of entrances as well as fastening Safety harness. The owner receives an alarm signal If an intruder ever come through the window and as soon as the IR-sensor detects an intruder.

Microcontroller is linked to an alarm via Bluetooth. It solves problems like utilizing a Tow truck by utilizing a limit switch, pressed upon signal is communicated to controller, finally order is sent to Dashboard through Bluetooth. Engine may be instructed by user as well as turn it off utilizing GSM for sending ruling signals.

Shruthi. K et al [7] implemented an anti-robbery system suitable for taking offender photograph as well as mailing it. which assistances in supervision as well as pursuing vehicles, which has been extended for utilization of car owners to empower them to take security contrary to robbery. This setup further enhances to perform as BlackBox of airplanes. Owner keeps position backup to decrease driver overtime, therefore enhances yield unique purposes for example passage of inmates or protected transport.

K. Kumar Jha [8,12] implemented IoT based oil monitoring system. This system compromises Temperature, Ultrasonic sensor and photodiode sensor with interfacing microcontroller and Arduino based programming of each sensor. The data obtained from various sample and analysis of each sample using ThingSpeak Channel a math work product. This paper explains details of sensor programming and extracting input and analysis of output data in real time. 


\section{OBJECTIVE AND METHODOLOGY}

An IoT-based vehicle theft detection framework is presented in this article. Since there's been many mechanisms used to locate a robbed car to date, the proposed technology overcomes the most inadequacies in existing systems as well as methods. As the dc motor begins, indicating that the car has been stolen, Arduino unlocks GPS, GSM and communicates alarm message to driver, and vehicle's location is posted using the IoT aided with Wi-Fi. For consumer comfort, whole system can be regulated by a switch. Figure 1 depicts the proposed machine block diagram.

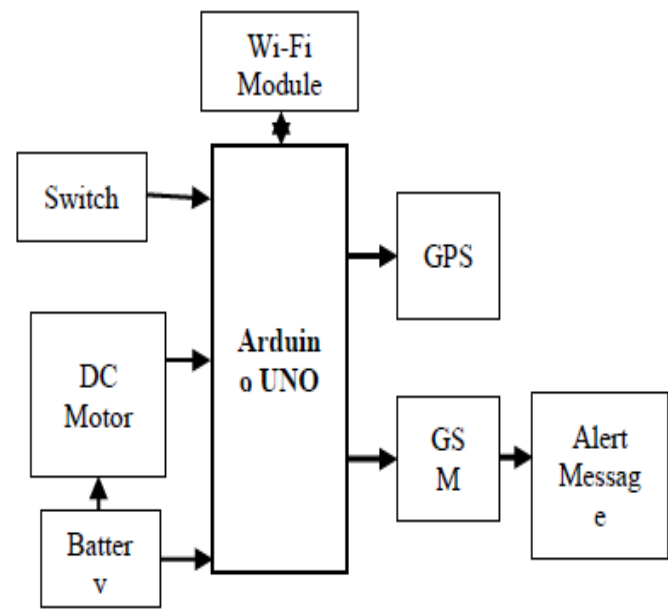

Figure 1. Anticipated System Block Diagram

\section{A. Arduino Uno:}

Microcontroller based on ATmega328 is the ArduinoUnoR3. There are fourteen optical input/output ports (among those 6 possibly be used for PWM), six analog-inputs, connector for USB, power port, crystal-oscillator of 16 $\mathrm{MHz}$, and ICSP-head which also includes reset switch in this board. By connecting to a device via battery or USB cable Arduino can be switched-on.

\section{B. Wi-fi Module-ESP-8266:}

ESP8266 is autonomous SOC which may provide connectivity to micro-controller or Wi-fi network. Each ESP8266 unit can be simply connected to an Arduino computer to gain Wi-Fi capability as it is pre-coded with AT-command set firmware. The ESP8266 unit is a lowpriced board with a large as well as rapidly growing community.

\section{DC Motor:}

Highest popular motor type. In most types, there are only two leads, positive as well as negative. It will spin when leads are linked to direct current source. engine spins in the opposite direction if the leads are interchanged with respect to power source.

D. Switch: This is used to turn on the fraud warning machine as a whole. As long as the ignition is turned on, an SMS warning with the vehicle's coordinates is sent as soon as the automobile begins. If it is turned-off, no message will be received, as well as no position information will be exchanged. We can monitor system activation and deactivation in this way by using switches.

E. GPS (Global-Positioning-System): It is a guidance system dependent on satellites. We use the NEO-6M GPS module since this works with a wide range of GPSreceivers. It has a ceramic antenna in built. It connects to a 3 Volt button-battery. In most cases, GPS operates in most of meteorological circumstances and in any location on the planet. To approximate 2D location, a GPS-receiver must be locked on to the minimal of three navigational satellites.

F. Global System for Mobile Communications: It is dedicated unit with SIM card and functions similarly to a cell mobile. We're using a SIM 900A (GSM) kit here. Dual Band GSM is used in the SIM900A modem.

\section{SYSTEM DESIGN:}

This segment, disuses the proposed individual use V-A-T system. This composes of the cloud IoT platform in the Internet, the theft detection device in the automobile, and mobile of owner. Figure 1, outlines system. Theftdiscovery module contains of GSM, microcontroller, and GPS. For the microcontroller, Arduino-Mega is implemented, here Android-Studio-IDE and Arduino-IDE are used in developing devices software package. It is available at low-price. Thing Speak Server a Iot platform implemented in which the basic functions for free.

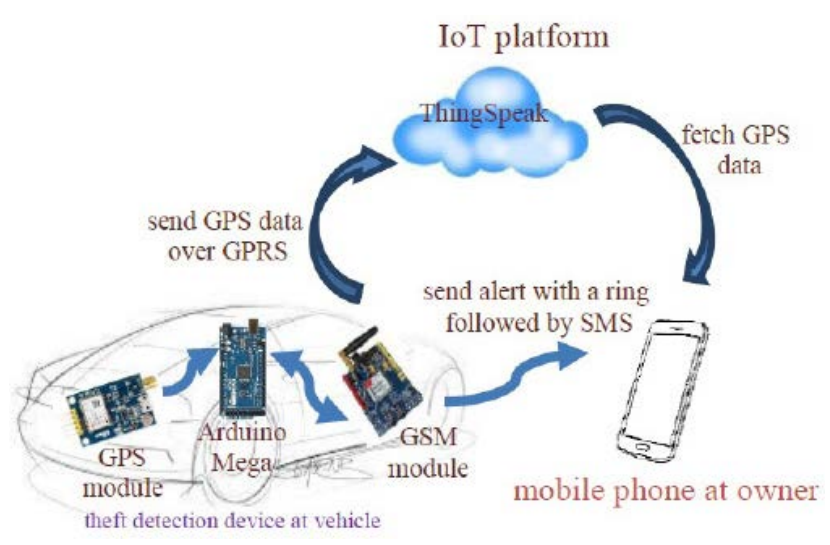

Figure 2. IoT Interface

The GPS contains theft-detection device which can be utilized to find theft. If the automobile position is monitored by GPS and altered without consent of owner more than threshold value, it is considered as theft. GSM 
uploads automobile location to cloud through Internetoccasionally, as well as transmitted the theft alarm message to the handset of the owner through SMS. Hardware portrayal Details of the adopted are described. Citation: Oo MZ, Paing SN, Funabiki N, Othman M (2019) A Personal Use Vehicle Anti-Theft Tracking System Using IoT Platform. Int J Computer Software Eng 4: 141. doi: https://doi.org/10.15344/2456-4451/2019/141

Microcontroller Arduino-Mega-2560 Microcontroller is utilized, which is founded on ATmega2560. Microcontroller contains 54 digital I/O pins (in which 15 is PWM), Sixteen analog-inputs, four UARTs hardwareserial-ports, $16 \mathrm{MHz}$ crystal-oscillator, power-jack, ICSPheader, USB-port, as well as restore-switch. It covers all the items required to sustain the microcontroller. Linking is done utilizing USB cable/power it with AC-DC adapter / battery to pc in order to start. GPS Ublox-Neo 7N-0-002 is utilized. It comprises of a peripheral antenna as well as embedded EEPROM. Needing a supply of 3-5 Volt as well as predefined baud rate $9600 \mathrm{bps}$. Supporting NMEA sentences. Neo-7N has a special feature of data logging, this aids in Realtime backup of coordinates, speed as well as time data to an internal 16Mbit-SQI-FLASH-memory. GPS receives info from navigational satellites then calculates topographical position. Upon powering up, NMEA sentence is sent out of a serial transmit pin at predefined baud rate that is 9600-bps.

SIMCOM produces GSM SIM900A which is used in this system. the frequencies of operation are 900 and 1800 $\mathrm{MHz}$, these 2 bands are automatically searched by SIM900A. Frequency range can also be set by AT command. AT command can be used to is configurable the baud rate from 1200-115200bps through. The GSM uses baud-rate of 9600bps for the theft detection module. With the use of TCP/IP stack, this unit is linked to Internet through GPRS. GSM SIM900A is a reliable wireless component and ultra-compact. It is a SMT type complete GSM/GPRS unit. it is economical as it is Design includes extremely-powerful one-chip processor including AMR926EJ-S core.

\section{a. Software description}

software adopted is described here. Thing Speak server is open-data platform and Application Programming Interface permits data collection, storage, analysis as well as visualization. It provides immediate visualization of posted data in device. With capability of executing MATLAB-code in ThingSpeak, with cloud processing as well as analysis of data. Thing Speak is utilized commonly in case of prototyping and proof of IoT idea, systems which are in need of analytics. Thing Speak facilitates users to visualize, analyze in addition aggregate data streams, real time from cloud. Data is stored in server, for process to be implemented it needs to create an account finally create a channel in that account. following which data streamed in may as well be observed in the respective fields. interaction between the microcontroller as well as modules which is used for sending and receipt of vehicle position data can be done using Arduino-IDE.

\section{b. Five-layer IoT architecture}

IoT goes beyond Internet-linking past conventional Devices like PCs, tablets, phones, and range of systems and day to day things that employ the embeddedtechnology to interact and interconnect by means of exterior environments. Key function of IoT is to gather and transform raw data into useful information. utilizing TCP/IP model, simplest IoT architecture has 3-layers. It consists of perception, network, as well as application layers [6].

Nevertheless, 5-layer is adopted as the 3-layer architecture is inadequate for the research of IoT, portrayed in Figure 3 [6]. Reaming layers perception as well as application, whereas the processing and transport layers are divided from network-layer. business-layer is the final-layer. perception-layer is used to gather surrounding data and identifies the objects. The information is moved through transport layer from perception-layer to processing-layer.

The processed data is storage, analysis, and processing done by processing-layer. The application-layer transports specific services to operators. The entire IoT system is managed by business-layer.

The perception-layer collects information of its surroundings and recognizes the things. Information is transferred through transport-layer from layer concerned with perception to processing. Layer concerned with processing backs-up, computes, and interprets moved content. operator obtains the specific services from the application layer. Whole IoT system manages businesslayer.

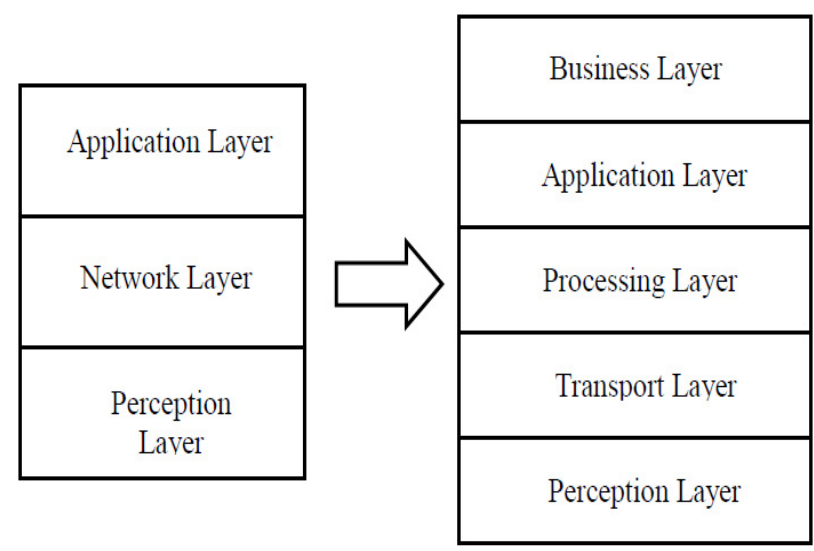

Figure 3: IoT-Three-to-Five-layer architecture. 


\section{c. IoT-architecture-of-proposes-system}

View-point of IoT 5-layers architecture, GPS works in perception, that gathers position info of automobile. GSM broadcasts, alarm to owner's handset by SMS, and transfers it between Thing Speak server and the microcontroller by utilizing the GPRS. Therefore, GSM/GPRS module remains answerable for the transport. position-data recognized by GPS is investigated at microcontroller and kept in Thing Speak server. Therefore, processing layer is answerable for the Thing Speak server and the microcontroller. An Android app is created for the end-user. It is responsible for layer of application. As it is for personal use, layer of business is not encompassed in this.

\begin{tabular}{|c|c|}
\hline $\begin{array}{l}\text { Business } \\
\text { Layer }\end{array}$ & Business Layer \\
\hline $\begin{array}{l}\text { Application } \\
\text { Layer }\end{array}$ & Android application \\
\hline $\begin{array}{l}\text { Processing } \\
\text { Layer }\end{array}$ & $\begin{array}{l}\text { Microcontroller, } \\
\text { ThingSpeak Server }\end{array}$ \\
\hline $\begin{array}{l}\text { Transport } \\
\text { Layer }\end{array}$ & GSM/GPRS \\
\hline $\begin{array}{l}\text { Perception } \\
\text { Layer }\end{array}$ & GPS \\
\hline
\end{tabular}

Figure 4: Five-layer architecture of proposed system.

\section{d. Device enactment}

The Arduino Mega microcontroller is linked to the GPS and GSM networks through a common ground. They get their energy from a battery or a power bank. The maximum functioning current of the Ublox-GPS-module at standard operating conditions is $40 \mathrm{~mA}$, according to the datasheet. The GSM-module transmits data at a rate of about 5 seconds per minute, using $200 \mathrm{~mA}$ on average, for a total of $24 \mathrm{~mA}$. The GSM module can consume 50 milliamperes. In the Arduino Mega, the Direct current for each I/O pin is 20 milliamperes. Approximate Amps drawn by unit is $(=40+24+50+20) 134 \mathrm{~mA}$ when the total current is added together.

Citation: Oo MZ, Paing SN, Funabiki N, An IoT-based vehicle anti-theft surveillance device for personal use.Int J Comput Softw Eng 4: 141. doi: https://doi.org/10.15344/2456-4451/2019/141 The battery capacity of the power bank used in the device is 13,000mAh. The battery's Amps rating is in milliamperes $(\mathrm{mA})$ as well as capacity in milliampere hours are used to calculate its life (mAh). Ratio of the battery capacity and product of exhausting rate as well as device ingestion represents the life of battery. The power bank would last about 82 hours if the consumption rate is assumed to be 0.7. The rate is 0.7 Allows for external factors that could have an effect on life of battery. Superior energy, energybank can supply, life is extended. Device's programmer is coded in $\mathrm{C}$ language then loaded to Arduino-Mega microcontroller utilizing the Arduino-IDE.

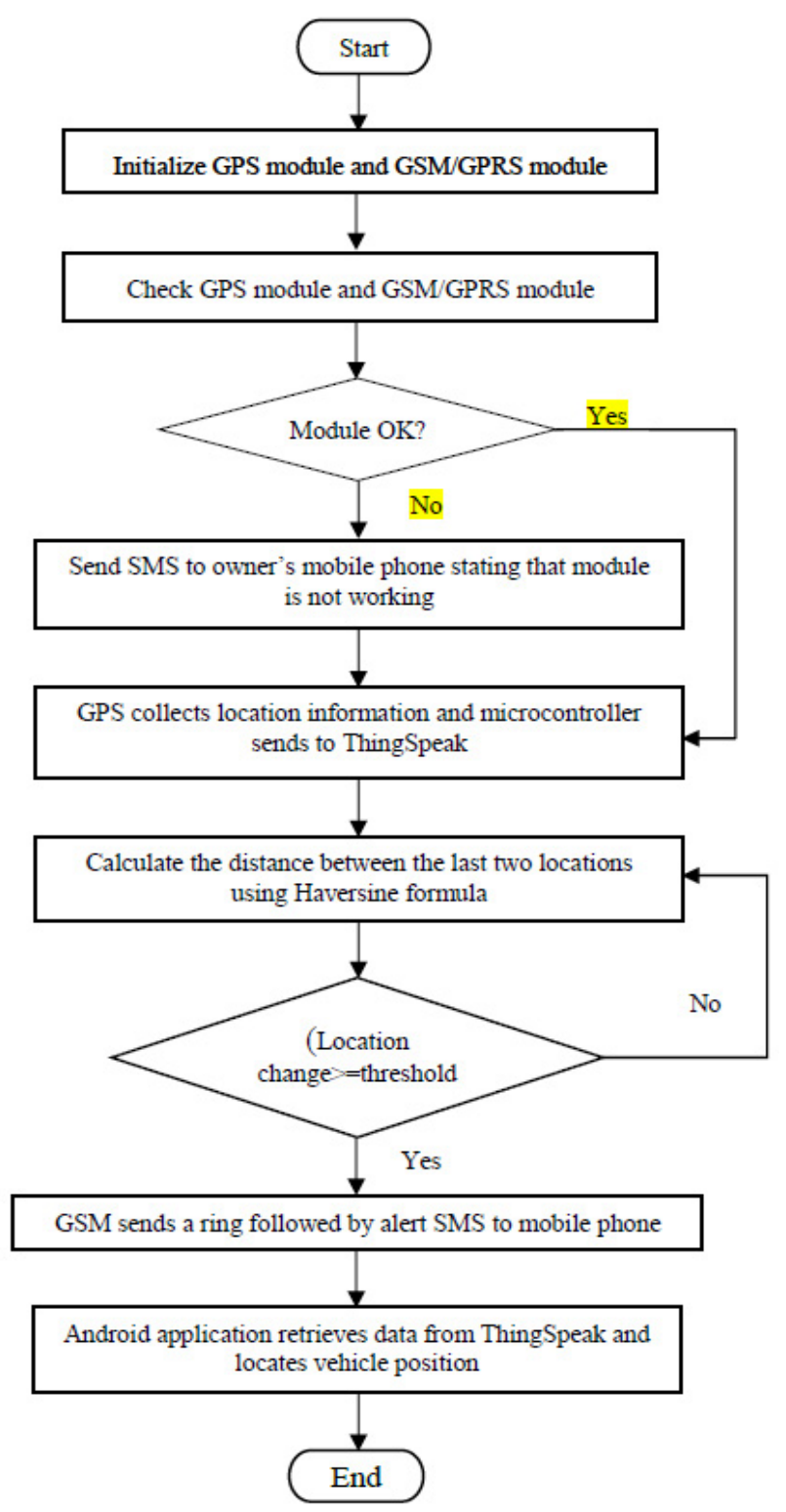

Figure 5: Flow Chart of Process

\section{CONCLUSION}

This presented paper gives very Cost effective and individual automobile anti-stealing pursuit utilizing cloud based plat-form named as Thing Speak, Adriano microcontroller, GPS module, and GSM module are used to create a vehicle anti-theft system. SMS is sent, warning the owner's phone. Trial applications were used to verify the accuracy of the applied functions. Future projects will include the expansion of Android apps for car owner program, system durability testing, and long-term assessment. 


\section{REFERENCE}

[1]. Motorbike numbers take off.

[2]. Thomas A (2018) List of 3 types of vehicle tracking system.

[3]. Shinde PA, Mane YB (2015) Advanced vehicle monitoring and tracking system based on Raspberry Pi. IEEE 9th Int Conf Intell Syst Cont.

[4]. Pandit AA, Mundra AK, Talreja J (2009) RFID tracking system for vehicles (RTSV). Int Conf Comput Intell Commun Syst Net.

[5]. Sadagopan VK, Rajendran U, Francis AJ (2011) Antitheft control system design using embedded system. IEEE Int Conf Vehi Electron Safety.

[6]. Sakthivel R, Dhinesh Babu R, Elstan B, Gopi Anand V, Aswin VA (2007) Vehicle Tracking and Locking System Based on Gsm and GPS. International Journal of Recent Trends in Engineering and Research 3: 213-219.

[7]. Sethi P, Sarangi SR (2017) Internet of Things: architectures, protocols, and applications. J Elect Comput Eng.

[8]. Keshav Kumar Jha, Rahul Arora, BS Pabla "Condition monitoring of lubricating oil Using internet of things (IoT)" had been reviewed by the board of international journal of Mechanical and production engineering research and development (IJMPERD); ISSN (online): 2249-8001; ISSN (print): 2249-6890; impact factor (JCC) (2019): 8.8746; index Copernicus value (ICV) - (2016): 60.6; Naas rating: 3.11; Vol - 10, issue 3; edition: June 2020"

[9]. Shruthi K, Ramaprasad P, Ray R, Naik MA, Pansari $\mathrm{S}$, et al. (2015) Design of an anti-theft vehicle tracking system with a smartphone application. Int Conf Inform Process.

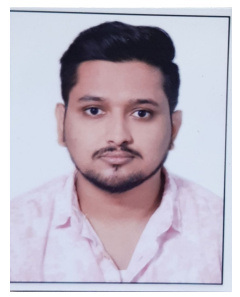

Utkarsh is M.Tech Scholar, Department of Mechanical Engineering , KCCITM Gr Noida ,UP, India. He had Completed B.Tech with from KCC Institute of Technology and Management, Gr Noida, UP, ,India.

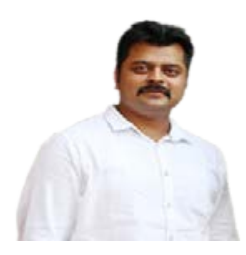

Mr. Keshav Kumar Jha is Assistant professor in Department of Mechanical Engineering, KCC Institute of Technology and Management, Greater Noida, UP. $\mathrm{He}$ is also working as Associate Dean of Student Welfare. Area of Specialisation is Manufacturing, Tribology, IoT, Sensor. Currently member of SAE India, IAENG.
[10]. Uddin MS, Ahmed MM, Alam JB, Islam M (2017) Smart anti-theft vehicle tracking system for Bangladesh based on Internet of Things. Int Conf Advan

Elect Eng.

[11]. Dey M, Arif MA, Mahmud MA (2017) Anti-theft protection of vehicle by GSM \& GPS with fingerprint verification. Int Conf Elect Compute Common.

Eng.

[12]. Kesahv Kumar Jha, BS Pabla “A Real Time Engine Oil Monitoring System for Diagnosis of Lubricant using IoT Network" was reviewed by experts in this research area and accepted by the board of 'Blue Eyes Intelligence Engineering and Sciences Publication' which has published in 'International Journal of Innovative Technology and Exploring Engineering (IJITEE)', ISSN: 2278-3075 (Online), Volume-9 Issue-8, June 19, Page No. 497-505.

[13]. Jian-Ming H, Guang-Hui L (2012) Automobile antitheft system based on GSM and GPS module. IEEE Int Conf Intell Net Intell Syst.

[14]. Salim K, Idrees IM (2013) Design and implementation of Web-based GPS - GPRS vehicle tracking system. IJCSET 3: 443-448.

[15]. Monawar T, Mahmud SB, Hira A (2017) Anti-theft vehicle tracking and regaining system with automatic police notifying using Haversine formula. Int Conf Advances in Elect Eng.

[16]. Rashed MAA, Oumar AA, Singh D (2013) A real time GSM/GPS based tracking system based on GSM mobile phone. Int Conf Future Gene Common Tech. 